\title{
Discours
}

Revue de linguistique, psycholinguistique et

informatique. A journal of linguistics, psycholinguistics and computational linguistics

$20 \mid 2017$

Varia

\section{Or, ora, maintenant : perspective temporelle et perspective argumentative}

Martin Becker et Marta Donazzan

URL : http://journals.openedition.org/discours/9290

DOI : 10.4000/discours.9290

ISSN : 1963-1723

Éditeur :

Laboratoire LATTICE, Presses universitaires de Caen

Référence électronique

Martin Becker et Marta Donazzan, «Or, ora, maintenant : perspective temporelle et perspective

argumentative », Discours [En ligne], 20 | 2017, mis en ligne le 22 septembre 2017, consulté le 19 avri 2019. URL : http://journals.openedition.org/discours/9290 ; DOI : 10.4000/discours.9290 

Revue de linguistique, psycholinguistique et informatique

\title{
Or, ora, maintenant : perspective temporelle et perspective argumentative
}

\author{
Martin Becker \\ University of Cologne \\ Marta Donazzan \\ Emerging Group «Dynamic Structure in Language and Communication» \\ University of Cologne
}

Martin Becker, Marta Donazzan, «Or, ora, maintenant: perspective temporelle et perspective argumentative», Discours [En ligne], 20 | 2017, mis en ligne le 22 septembre 2017.

URL: http://discours.revues.org/9290

Titre du numéro: Varia

Coordination: Shirley Carter-Thomas \& Laure Sarda 



\title{
Or, ora, maintenant : perspective temporelle et perspective argumentative
}

\author{
Martin Becker \\ University of Cologne \\ Marta Donazzan \\ Emerging Group «Dynamic Structure in Language and Communication » \\ University of Cologne
}

Nous présentons dans cet article une analyse contrastive de la distribution et de l'interprétation de trois adverbes, or et maintenant du français et ora de l'italien. Notre objectif est de mettre en évidence le parallélisme entre l'emploi temporel et l'emploi argumentatif de ces adverbes, et de souligner les différences entre l'utilisation de ora en italien et de or/maintenant en français. Nous défendons l'idée que ora et or/maintenant réalisent la même fonction sémantique, celle d'actualiser le point de perspective du récit. Nous avançons l'hypothèse que le trait sémantique qui justifie cette fonction est le lien essentiel entre la lecture temporelle et épistémique. Ce lien est le résultat d'un procès d'abstraction de ces adverbes temporels et de leur utilisation comme marqueurs sur le plan discursif.

Mots clés: adverbes temporels, marqueurs discursifs, structure temporelle, argumentation, point de perspective, point de référence

This paper presents a contrastive analysis of the distribution and interpretation of the French adverbs or and maintenant and the Italian adverb ora. Our goal is to describe the parallelism between the temporal and argumentative use of these three adverbs, and to underline at the same time the differences in the use of ora with respect to or / maintenant. We claim that ora and or / maintenant share a common function, which is to update the point of perspective in the temporal structure of the ongoing discourse. Next, we propose that the semantic feature that justifies this updating function is the essential link between the temporal reading and the epistemic reading, which can be considered as the typical result of a process of abstraction of temporal adverbs into discourse connectives.

Keywords: temporal adverbials, discourse connectives, temporal structure, argumentative structure, perspective point, reference point

Remerciements: Nos remerciements vont à l'université de Cologne et à la DFG (Deutsche Forschungsgemeinschaft), qui ont rendu possible cette étude à travers le support de l'Emerging Group «Dynamic Structure in Language and Communication» et du Sonderforschungsbereich 1252 «Prominence in Language». Nous sommes aussi redevables aux nombreux collègues qui participent à ces projets collaboratifs pour leur aide et pour leurs encouragements.

\section{Introduction}

L'objet de cet article est d'étudier la sémantique de trois mots qui sont employés à différents niveaux du discours, et d'expliquer le rapport qui existe entre leurs différents emplois. Nous commençons par nous intéresser aux adverbes italien ora et français 
maintenant, qui peuvent être décrits à travers deux emplois principaux: celui d'adverbe temporel comme en [ra-b], et celui de marqueur discursif comme en [2a-b].

[I] a. Aujourd'hui to novembre je commence mon journal. Cela me paraît un peu bête quand je pense que je fais comme les petites filles d'autrefois. Mais je suis isolée maintenant à cause de la guerre et je suis obligée de confier mes peines à quelqu'un, eh bien ce quelqu'un ce sera mon cahier.

(Frantext: D. Domenach-Lallich, Demain il fera beau: journal d'une adolescente (novembre 1939-1944), Lyon, Éditions Bga Permezel, 200I, p. 43)

b. Guardò altrove e disse con grande serietà:

- Ora è un po' difficile! Ma ci penserò! Che cosa sa fare Lei?

- Essa sapeva leggere, scrivere e far conti. Di lingue non conosceva che il triestino e il friulano.

(CORIS: I. Svevo, Novella del buon vecchio e della bella fanciulla [1929], Milan, A. Mondadori, 2004)

Il regarda ailleurs et dit très gravement:

- ORA c'est un peu difficile! Mais je vais y réflécbir! Que savez-vous faire?

- Elle savait lire, écrire et calculer. Elle ne connaissait d'autres langues que le triestin et le frioulan'.

[2] a. Julie et Marcel se voient souvent ce temps-ci. Maintenant, ça ne veut pas dire qu'ils sortent ensemble.

(Nef 1980: 156)

b. In una democrazia rappresentativa, i politici sono per definizione una «classe aperta», con uscite ed entrate frequentissime. [...] Ora, nessuno in buona fede può affermare che i politici in Italia siano una classe aperta e che le entrate e le uscite, cioè il rinnovamento del personale, sia soddisfacente.

(E. Scalfari, La Repubblica, 1986)

Dans une démocratie représentative, les politiciens sont, par défnition, une «classe ouverte», avec des sorties et des entrées très fréquentes. [...] ORA, personne ne peut affirmer en toute bonne foi que les politiciens en Italie sont une classe ouverte, et que les entrées et sorties, c'est-à-dire le renouvellement du personnel, sont satisfaisantes.

Tout en ayant une distribution assez similaire, ces deux adverbes présentent aussi des différences qui concernent à la fois leur emploi d'adverbe temporel et leur statut de marqueur discursif. Tout d'abord, maintenant et ora présentent des différences de distribution dans leur utilisation en tant qu'adverbe temporel. En particulier, alors qu'il a été plusieurs fois souligné que maintenant n'apparaît qu'avec les temps de l'imparfait ou avec des prédicats statifs (Nef, I980; Kamp et Rohrer, I983; Jouve, 1990; De Mulder, 2006), ora peut être utilisé aussi avec des temps perfectifs, et notamment avec le passé simple (passato remoto) (voir l'exemple [3]).

1. Sauf mention contraire, nous avons réalisé les traductions de l'italien vers le français des exemples. 
[3] Guido seppe disarmarmi subito. Avevo tenuto sinora il segreto, ma ora, con un abbandono da buon ragazzo, mi disse ogni particolare di quei suoi affari.

(CORIS : I. Svevo, La coscienza di Zeno [1923], Turin, Einaudi - Gallimard, 1993)

Guido sut me désarmer tout de suite. J'avais gardé le secret jusqu'à présent, mais ORA, avec un abandon de bon garçon, il me raconta tous les détails de ses affaires.

En ce qui concerne l'emploi argumentatif (c'est-à-dire comme marqueur discursif), maintenant entre en concurrence en français avec or, qui vient, comme l'italien ora, de l'étymon latin hora. Dans les deux langues, les formes or/ora ont évolué, selon un processus de grammaticalisation, au-delà de leur statut lexical vers la fonction de marqueur discursif, ou connecteur du discours. En effet, or en français moderne est un connecteur à part entière (Bertin, 200I), et ses contextes d'utilisation dépassent largement ceux de maintenant dans cette même fonction.

Il existe un certain nombre d'études formelles portant sur les adverbes temporels comme ora et maintenant, qui ont été développées notamment dans la tradition anglo-saxonne autour de l'adverbe anglais now. Ces études se sont concentrées cependant exclusivement sur l'analyse de now en tant qu'adverbe temporel dans le discours (Kamp, I97I et 20I3; Altshuler, 2013; Hunter, 20I2). En ce qui concerne les langues qui font l'objet de notre travail, alors qu'il existe de nombreuses analyses portant sur le français (Nef, I980; De Mulder, 2006), il n'existe pas, à notre connaissance, d'étude spécifique de ora en italien. Notre but dans ce travail est donc de développer une analyse sémantique comparée de maintenant et de ora en italien, qui puisse expliquer d'un côté le lien entre les deux emplois, temporel et argumentatif, que ces deux adverbes partagent, et, de l'autre, les différences que nous avons observées. Pour constituer une base empirique, nous nous sommes appuyés principalement sur l'analyse qualitative d'un choix de textes contemporains, appartenant aux genres littéraire et journalistique ${ }^{2}$. Le choix de ces deux genres textuels est motivé principalement par les raisons suivantes. D'un côté, maintenant/ora, dans leur emploi temporel, peuvent être rapprochés des adverbes repérant le temps de l'énonciation, qui ont été au centre des études portant sur la définition du récit (Banfield, 1982); ce genre littéraire se prête de manière privilégiée à une étude distributionnelle de ces formes et à la saisie de leur valeur sémantique. De l'autre côté, la discussion politique dans les textes journalistiques nous offre un contexte idéal pour étudier l'emploi de or/ora dans leur fonction de structuration et d'orientation d'un texte argumentatif.

2. Pour le français, nous avons considéré les articles du Monde de l'année 2002, et nous avons sélectionné dans la base Frantext des récits publiés entre i96o et la fin des années 2000. Pour l'italien, nous avons analysé des textes littéraires allant du début du XXe siècle aux années 2000, tirés du corpus CORIS (http://corpora.ficlit.unibo.it/coris_eng.html), et les articles du quotidien La Repubblica compris entre les années I980 et 2000, tirés du corpus «La Repubblica» (http://sslmit.unibo.it/repubblica). Nous avons extrait un total de III exemples attestés dans Le Monde de l’année 2002, et nous avons analysé de plus près les so premiers d'entre eux. Parallèlement, nous avons extrait environ roo exemples dans le corpus «La Repubblica», entre les années 1980 et 2000 , et nous avons analysé plus spécifiquement 40 d'entre eux. 
L'article est organisé comme suit. Tout d'abord, nous allons considérer l'analyse temporelle de ora/maintenant. En nous appuyant sur l'analyse avancée originairement par Kamp (20I3), nous proposerons que ora/maintenant sont des adverbes qui agissent sur l'organisation temporelle du récit ou du discours en modifiant la perspective dans laquelle les événements sont présentés. Dans ce sens, comme le note aussi Altshuler (2013) dans son analyse de l'adverbe now de l'anglais, ora/maintenant sont «transparents» aux relations qui font progresser la narration, c'est-à-dire qu'ils ne font pas progresser le temps de référence (TR). La contribution de ces adverbes est celle d'actualiser (update) la perspective, en indexant le point de perspective (PP) à un repère saillant dans le discours. On peut considérer qu'il existe trois contextes principaux dans lesquels ce changement de perspective a lieu : le discours indirect (DI), le discours indirect libre (DIL) et le contexte de récit et de narration (RN). Nous verrons que dans tous ces cas, l'utilisation de ora/maintenant peut s'expliquer par l'introduction d'un PP interne au récit. L'analyse de ces cas nous permettra enfin d'aborder la question des différences aspectuelles observées relativement à la distribution de ora/maintenant, et notamment la question de la compatibilité de ora avec le passé simple.

Dans la deuxième partie de ce travail, nous aborderons l'analyse de ora/maintenant en tant que marqueurs discursifs, et nous montrerons que le trait d'actualisation que nous avons postulé pour ora/maintenant sur le plan propositionnel peut être étendu à l'emploi de ces adverbes dans la structuration et l'orientation argumentative du discours.

\section{Ora/maintenant temporels}

\subsection{Limites de l'analyse déictique}

À première vue, les adverbes temporels comme ora et maintenant peuvent être rapprochés d'expressions déictiques ancrées sur le moment de l'énonciation, comme aujourd'bui ou demain. Cela semble être l'analyse la plus pertinente pour ces adverbes quand ils entrent en co-occurrence avec le présent de l'indicatif, comme dans les exemples [ra-b] proposés dans la section précédente. Cependant, une définition de ora et maintenant comme adverbes déictiques n'est pas sans poser problème.

La sensibilité au contexte propre aux expressions déictiques est le plus souvent décrite, pour ce qui est des analyses formelles, en suivant l'analyse proposée par Kaplan (1989), analyse dont nous donnons un bref aperçu ci-dessous. Supposons que Marie et Jeanne prononcent la même phrase à deux moments distincts $t_{I}$ et $t_{2}$, correspondant, par exemple, au lundi et au mardi de la même semaine.

\section{[4] a. Marie à $t_{1}$ (lundi): «Maintenant il fait beau» \\ b. Jeanne à $\mathrm{t}_{2}$ (mardi) : «Maintenant il fait beau»}

Marie et Jeanne prononcent la même phrase, mais elles disent deux choses différentes: Marie dit qu'il fait beau lundi, tandis que Jeanne affirme qu'il fait beau mardi. Supposons que le lundi en question il fasse beau, mais que Jeanne se trompe, et que le mardi il soit en train de pleuvoir. Autrement dit, dans ce cas de figure, 
[4a] est une proposition vraie, et [4b] est fausse. Les deux phrases correspondant aux énoncés [4a] et [4b] doivent être ainsi distinguées sur la base de la valeur de vérité des propositions qu'elles expriment. Kaplan (1989) rend compte de cette sensibilité au contexte d'énonciation en introduisant une distinction entre deux types de sens, le caractère d'une expression et son contenu. Le caractère d'une expression déictique est fixé par convention : le caractère de maintenant, par exemple, est celui de dénoter le moment d'énonciation. Dans la théorie de Kaplan, les expressions indexicales comme maintenant n'ont cependant de contenu qu'en relation à un contexte. Chaque contexte $c$ est associé à un agent, un indice temporel, un indice spatial et un monde possible. En relation avec un contexte $c_{l}$, le caractère de maintenant attribue donc comme contenu à cette expression l'indice temporel de $c_{r}$. Ainsi, la phrase «Maintenant il fait beau» a des contenus différents qui varient avec le contexte dans lequel elle est énoncée: dans le contexte $c_{I}$ de lundi, la phrase exprime une proposition vraie, tandis que dans le contexte $c_{2}$ de mardi, quand elle est énoncée par Jeanne, elle exprime une proposition fausse. Dans notre exemple [I], le caractère de maintenant attribue comme contenu à l'expression, l'indice temporel correspondant au Io novembre 1939 .

Cette analyse déictique est toutefois mise à l'épreuve, dans le cas de ora et maintenant, par de nombreuses occurrences de ces adverbes où l'indice temporel ne peut être identifié aisément au temps d'énonciation. Nous pouvons distinguer principalement trois cas de figure.

Le premier est celui du DI. Celui-ci est caractérisé par la présence explicite d'un locuteur interne, auquel est attribuée l'assertion représentée par la phrase pertinente. Cette phrase est généralement subordonnée à un verbe de communication, comme dans les exemples [5] et [6]. Dans ces exemples, maintenant et ora co-occurrent avec l'imparfait de l'indicatif, qui peut être analysé comme un présent dans le passé du locuteur fixé par le récit (Giorgi et Pianesi, 2004).

[5] Les choses s'étaient gâtées devant la tombe parce que Patrice, agacé lui aussi par le discours du diacre et ce qu'il considérait comme des simagrées bondieusardes, avait dit que maintenant chacun pouvait faire ses adieux à Juliette de la façon qui lui convenait.

(Frantext: E. Carrère, D'autres vies que la mienne, Paris, POL, 2009, p. 206)

[6] Larry gli disse che era tutto a posto, che ora se ne poteva andare, e lasciò che andasse avanti e poi gli sparò mentre Eddie si chinava per uscire strisciando dal cunicolo.

(CORIS : S. Deane, Le parole della notte, V. Mantovani (trad.), Milan, G. Feltrinelli, 1997)

Larry lui dit que tout allait bien, que ORA il pouvait partir, et le laissa avancer et ensuite le buta, pendant qu'Eddie se penchait pour sortir du tunnel en rampant.

Un type particulier de DI est représenté par le DIL. Ce mode du discours, qui est propre au récit littéraire, se rapproche du DI dans la mesure où, dans ce cas 
aussi, l'auteur attribue les propositions exprimées par les phrases pertinentes à un personnage interne au récit. Comme il a été plusieurs fois noté ${ }^{3}$, le DIL se démarque cependant du DI sur le plan formel. Tout d'abord, le DIL n'est pas constitué de phrases subordonnées à des verbes de communication. En outre, le DIL peut être constitué de phrases indépendantes du point de vue illocutoire, comme les phrases exclamatives et interrogatives dans l'exemple italien [7]. Deuxièmement, ce contexte permet une utilisation beaucoup plus libre des expressions déictiques, qui ne se limitent pas à ora et maintenant. Comme on peut le remarquer notamment dans l'exemple français proposé sous [8], le DIL permet l'utilisation d'un éventail plus étendu d'expressions déictiques, exemplifiées dans ce cas par les démonstratifs cet [état], ce [jour]/cette [nuit].

[7] Camminò per parecchio tempo senza meta, per tranquillarsi. Forse Amalia aveva veduto bene e il suo abbandono era stato per Angiolina la piú energica delle educazioni. Forse ella lo amava ora! Camminando fece un sogno delizioso. Ella lo amava, lo seguiva, s'attaccava a lui, ed egli continuava a fuggirla, a respingerla. Quale soddisfazione sentimentale!

(CORIS: I. Svevo, Senilità [1898], Milan, Dall'Oglio, 1971)

Il marcha pendant longtemps sans se donner un but, pour se calmer. Peut-être Amalia avait-elle vu juste et son abandon avait été pour Angiolina la plus énergique des éducations. Peut-être qu'elle l'aimait ORA! Tout en marchant, il fit un rêve délicieux. Elle l'aimait, le suivait, s'attachait à lui, et lui, il la fuyait, la repoussait sans cesse. Quelle satisfaction sentimentale!

[8] Elle avait un accès de réalisme insupportable pour m'avoir vue dans cet état, cauchemardant cette nuit et maintenant frappée d'anesthésie générale, par sa faute, disait-elle, en ce jour qui fêtait l'Enfant et sa sainte famille.

(Frantext: A.-M. Garat, Les mal famées, Arles, Actes Sud, 2000, p. 16I)

Intuitivement, on pourrait imaginer que les contextes du DIL et du DI ne posent pas de problèmes particuliers à une analyse déictique de ora et maintenant, car on pourrait simplement postuler un décalage du contexte du narrateur par rapport au contexte du locuteur interne au récit, et maintenir une analyse du contenu des déictiques par une fonction du contexte au moment d'énonciation. Cette solution, qui peut convenir pour les déictiques pour ainsi dire "purs» (Schlenker, 2004; Sharvit, 2008), s'avère cependant problématique quand on considère un troisième cas où maintenant/ora peuvent apparaître avec un temps du passé. Il s'agit des contextes dans lesquels on ne retrouve aucun des traits formels du DI ou du DIL, et qu'on appellera simplement ici, en opposition à ces deux derniers cas, des contextes de RN. Deux exemples pertinents sont présentés en [9] et [io] ${ }^{4}$.

3. Voir Giorgi (2015) et Guéron (2015) pour des analyses récentes.

4. Un de nos relecteurs nous fait remarquer que le passage sous [Io] pourrait tout aussi bien être interprété comme un cas de DIL, où ora marquerait le présent déictique du personnage interne au récit. Nous 
[9] Parce que Marie était en grande colère, elle n'avait plus son regard de lavande mouillée. Maintenant, elle jetait des yeux furibonds de côté et plumait dans son tablier les cailles avec férocité, avec méchanceté, toute pâle, énervée, échevelée.

(Frantext: A.-M. Garat, Les mal famées, Arles, Actes Sud, 200o, p. I49)

[ı] L'atto legale, che stava preparando l'avvocato, le piaceva molto e l'appagava. Abituata sempre ad obbedire e poi trattata peggio d'una serva dal marito, ora anche lei aveva un avvocato che pensava ai suoi interessi; ed era impazientissima che l'atto fosse completato.

(CORIS : F. Tozzi, Il podere [192I], Milan, Rizzoli, 1983)

L'acte légal que l'avocat était en train de préparer lui plaisait beaucoup et la comblait. Habituée comme elle l'avait toujours été à obéir et puis traitée pire qu'une esclave par son mari, ORA elle aussi avait un avocat qui s'occupait de ses intérêts; et elle attendait avec impatience que cet acte fût terminé.

Employés dans le RN, maintenant/ora s'opposent aux démonstratifs et aux adverbes temporels déictiques comme aujourd'bui, car ces derniers ne sont pas admis dans ce contexte. Pour autant, tous les contextes RN ne légitiment pas leur utilisation. Dans la prochaine section, nous allons nous concentrer d'abord sur ce troisième type de contexte (RN), et nous reviendrons ensuite sur le DI et le DIL.

\subsection{Contribution de ora/maintenant dans le RN et le DIL}

La contribution de maintenant/ora temporels dans le RN ne peut être évaluée sans que soient prises en compte les contraintes qui régissent la distribution de ces adverbes dans ce type de texte narratif. Ces dernières peuvent être résumées par deux caractéristiques principales, que nous reprenons ci-dessous:

- premièrement, dans la littérature concernant l'adverbe français maintenant, il a été noté à plusieurs reprises (Nef, I980; De Mulder, 2006) que cet adverbe temporel est utilisé presque exclusivement avec des prédicats imperfectifs, et, plus précisément dans les contextes RN, avec les temps de l'imparfait ${ }^{5}$. Pour comprendre les conséquences de cette préférence distributionnelle, nous allons faire un bref détour, dans la section 2.2.I, sur la sémantique de l'imparfait dans les langues romanes, en nous concentrant en particulier sur son opposition au passé simple dans la construction du récit;

sommes d'accord pour reconnaître que la distinction entre DIL et RN est dans certains cas très floue, c'est pourquoi nous avons pris comme critères décisifs pour l'appartenance au DIL les traits formels évoqués plus haut, et notamment la présence de phrases indépendantes du point de vue illocutoire et d'un éventail plus étendu d'expressions déictiques. Nous avons donc décidé de classer le texte en [Io] parmi les exemples de RN précisément parce qu'il ne présente aucun de ces traits.

5. Cela est vrai aussi pour la plupart des exemples en italien, même si, comme nous l'avons déjà souligné dans la section d'introduction, l'adverbe ora en italien peut être associé plus facilement à des temps perfectifs. Nous reviendrons sur cette particularité dans la section 2.2.I. 
- deuxièmement, cette distribution peut s'expliquer par la particularité des adverbes comme maintenant/ora à ne pas interagir avec la structuration du récit, c'est-à-dire avec l'enchaînement temporel des événements tel qu'il est exprimé par le choix des temps verbaux et d'autres expressions adverbiales. En revanche, nous proposons d'attribuer à maintenant/ora un contenu lexical qui implique un potentiel de changement sur le plan de la présentation de l'information.

Notre analyse s'articule autour de deux propositions, qui seront l'objet de la section 2.2.2. D'un côté, nous proposons d'opérer une dissociation entre deux repères temporels : un repère temporel qui organise la progression du récit (le TR) et un repère temporel qui représente plus particulièrement le rapport entre le TR et le temps d'énonciation dans le système originaire de Reichenbach. Suivant Kamp (2013), nous appellerons ce repère le PP. De l'autre côté, nous proposons que la fonction de maintenant/ora est celle d'indexer de façon anaphorique (ou co-indexer) le PP au TR le plus saillant dans le contexte. En d'autres termes, dans notre analyse, maintenant/ora sont transparents vis-à-vis des relations sur le plan du récit, et notamment pour ce qui est de la relation entre les événements et le TR; la fonction de ces adverbes est celle d'apporter une contribution sur le plan de la perspective temporelle, en introduisant une mise à jour (update) du PP. Si maintenant/ora ne jouent aucun rôle sur le plan du récit (d'où leur utilisation privilégiée avec les temps anaphoriques comme l'imparfait), ils apportent néanmoins une contribution, en signalant un repère pertinent dans le système des relations temporelles sur le plan de la perspective. Notons enfin que l'introduction d'un paramètre de saillance nous permet de rendre compte non seulement de la contribution de maintenant/ora dans le RN, mais aussi de leur distribution dans les contextes de DIL.

\subsubsection{Distribution avec les temps verbaux. Utilisation avec l'imparfait et transparence dans le récit}

Dans un modèle qui veut intégrer l'information temporelle exprimée au niveau propositionnel avec la construction du discours, la contribution des temps du passé peut être représentée à travers des contraintes formelles. La définition donnée sous [II], dans laquelle nous adaptons le traitement formel proposé par Kamp et Rohrer (1983), représente la contribution temporelle du passé simple en français: dans [ıIa] l'événement $e$ décrit par le prédicat au passé simple est proprement inclus dans un intervalle $\left(t_{l o c}\right)$ qui constitue le temps de localisation de $e$; dans [IIb] le temps de localisation précède $(<)$ sur l'axe temporel le temps d'énonciation $(n)$; dans [IIc] l'interprétation du passé simple dans le cadre de l'ordre temporel du discours est obtenue en prenant le temps de localisation de l'éventualité antérieure $\left(t_{\text {loc- } t}\right)$ comme point de repère. Ce dernier point de repère est le TR sur le plan du discours; au niveau discursif, la contribution du passé simple consiste donc à exprimer le rapport entre le point de localisation de l'éventualité et le TR comme un rapport de succession. En d'autres termes, le passé simple introduit un point de localisation et actualise le TR, en contribuant, de cette manière, à la dynamique événementielle et donc à la «séquentialisation» du discours. 
[II] a. e $\subseteq \mathrm{t}_{\text {locx }}$

b. $\mathrm{t}_{\text {locx }}<\mathrm{n}$

c. $t_{\text {locx }}>t_{\text {locx-1 }}(=T R)$

L'imparfait en français signale en revanche que l'éventualité en question n'introduit pas un nouveau temps de localisation, mais doit s'ancrer à un repère temporel déjà introduit dans le contexte. Dans ce sens, l'imparfait est un temps anaphorique, qui rapporte l'éventualité à un TR déjà présent dans le contexte et, de ce fait, ne contribue pas à l'avancement de la dynamique événementielle. Sous [I2a], ce rapport est défini comme un recouvrement (O) entre l'événement et le TR.

\section{[I2] a. e O TR \\ b. $\mathrm{TR}<\mathrm{n}$}

L'imparfait n'introduit pas de nouveau repère temporel dans le récit. Cette caractéristique a également été observée pour ora/maintenant (Kamp et Rohrer, 1983; Smith, 199I et 1997; Altshuler, 20Iob). Il est possible d'apprécier cette particularité de ora/maintenant en considérant précisément l'occurrence de ces adverbes avec des prédicats conjugués aux temps du passé. Une phrase isolée à l'imparfait ou au passé simple est perçue comme incomplète en français (exemples [iza-b]) ${ }^{6}$, et elle n'est jugée comme étant bien formée que si une expression temporelle explicite fournit un point d'ancrage temporel pour localiser l'événement décrit, comme le montrent les exemples [13c-d] vis-à-vis du passé simple. On peut ainsi voir que maintenant ne permet pas de localiser le temps d'assertion passé dans le récit (Smith, 1977; Altshuler, 20I2), et qu'il s'oppose en ce sens aux adverbiaux temporels qui introduisent un repère temporel explicite, comme c'est le cas pour l'adverbial en juin 1934.

[13] a. \#On construisait un immeuble.

b. \#On construisit un immeuble.

c. \#On construisit maintenant un immeuble.

d. En juin 1934, on construisit un immeuble.

Examinons maintenant un exemple attesté. Dans l'extrait de RN de l'exemple [I4], la progression narrative est donnée par le verbe au passé simple (offrit), qui introduit un TR dans le récit. L'événement d'habiter dans la caserne, exprimé par un prédicat à l'imparfait de l'indicatif, est donc interprété comme étant au moins co-extensif avec ce TR. On voit bien que l'adverbe maintenant ne contribue pas à la progression textuelle: la suppression de maintenant, dans l'exemple manipulé sous [I5], ne modifie pas l'agencement des événements dans le récit.

6. Mais une énonciation «out of the blue» est possible avec le passé simple, voir des énoncés comme enfin vint Malberbe/Pierre frappa à la porte, qui sont acceptables au début d'un roman. 
[14] Clara, apitoyée, m’offrit alors pour trois sous «sa» chambre de service, dans la caserne 1935 qu'elle habitait maintenant rue Tournefort.

(Frantext: F. Nourissier, À défaut de génie, Paris, Gallimard, 2000, p. 239)

[I5] Clara, apitoyée, m'offrit alors pour trois sous «sa» chambre de service, dans la caserne I935 qu'elle habitait rue Tournefort.

Dans le cadre de notre analyse, la question qui se pose est naturellement la suivante: s'ils n'introduisent pas de localisation temporelle pour l'événement, quelle est donc la contribution sémantique de ora/maintenant? Dans le cadre du formalisme de la «théorie de la représentation du discours» (Discourse Representation Theory-DRT), Altshuler (2014) répond à cette question en proposant que l'adverbe anglais now, qui comme maintenant n'introduit pas de nouveau repère dans le discours, possède en revanche un contenu anaphorique, et permet de trouver une interprétation temporelle pour saturer l'argument de temps dans le contexte ${ }^{7}$. Selon Altshuler (20I4), now recentre la perspective temporelle en renvoyant explicitement au repère temporel qui lui sert d'antécédent; en d'autres termes, now «rappelle» le point où le récit avait été laissé ("pick up the narrative where it left off»; Altshuler, 20I2: 82), par exemple après une digression. Cette définition rend compte du caractère anaphorique de now, mais ne permet pas à elle seule d'expliquer les restrictions observées vis-à-vis de son interprétation et les effets pragmatiques de saillance qui découlent de son utilisation. Rappelons en effet que tous les contextes de RN ne permettent pas l'utilisation de maintenant/ora. Afin de donner une réponse à notre question de départ, par la suite nous examinerons donc de plus près la contribution de maintenant/ora dans le RN.

Pour conclure cette section dédiée à la distribution des adverbes, attardonsnous brièvement sur les différentes restrictions qu'on peut observer en français en comparaison avec l'italien.

Nous avons vu qu'en français, maintenant s'emploie presque exclusivement avec l'imparfait, alors qu'en italien, ora apparait souvent dans le contexte d'un temps perfectif du passé (le passato remoto).

Par souci de précision, notons qu'il existe de rares cas, discutés notamment par Nef (1980) et De Mulder (2006), où maintenant apparaît avec un passé simple en français. Ces contextes semblent être limités à la co-occurrence avec des verbes phasiques qui, comme commencer dans l'exemple [ı6b] (tiré de Nef, 1980: 148), décrivent une éventualité dont le point initial coïncide avec le point de repère de maintenant.

7. Dans le cade de la DRT, Altshuler (20I4) adopte cette définition pour l'adverbe currently, qu'il rapproche explicitement de now (discuté en Altshuler, 20Iob). Une analyse différente de l'adverbe anglais now, dans laquelle cet adverbe est également traité comme un opérateur anaphorique, est proposée par Hunter (2012). Hunter considère now comme un déictique qui possède un contenu présuppositionnel, qui est mis en avant dans les contextes de récit. Selon cette analyse, now introduit une présupposition d'existence pour un indice temporel qui doit être identifié avec un référent du discours, un temps déjà disponible dans le contexte. 
[I6] a. \#Maintenant, les couvertures le génèrent.

b. Maintenant, les couvertures commencèrent à le gêner.

(Nef, I980: I48)

Dans l'exemple [ig], c'est à travers le redressement de la perspective opéré par ora que le locuteur met l'accent sur la victoire d'Octavien. Il s'agit donc d'un mécanisme

D'après l'analyse de corpus de Noël (1996), maintenant peut apparaitre aussi avec le passé simple de verbes d'état psychologique. Ces prédicats peuvent aussi être analysés comme des verbes phasiques, qui marquent le point initial d'un processus psychologique. Considérons par exemple l'extrait suivant (cité par De Mulder, 2006: 27), où maintenant marque le début des deux états psychologiques exprimés par le passé simple de croire et de sentir.

[17] Certes, il avait pu s'imaginer par avance quels pourraient être - quels ne pourraient pas être - les sentiments de Heide pour Albert, mais indépendamment d'une curiosité peut-être seulement maladive, il crut deviner maintenant à sa conduite un plus déroutant motif, dont l'éclat retentit dans son cerveau avec les élancements de la fièvre. [...] et il sentit maintenant - et la pleine conscience lui sembla battre son front comme l'aile même de la folie - qu'il l'avait lui-même amenée à Albert pour la plonger au sein de leur vie double [...].

(Frantext: J. Gracq, Au Château d'Argol [1938], Paris, Corti, 1946, p. 35)

En fait, cette préférence distributionnelle peut être étendue à ora en italien, qui, certes, peut entrer en co-occurrence avec un temps perfectif, mais admet uniquement, dans ce contexte, la présence de verbes phasiques. En italien aussi, il est toujours possible d'utiliser ora avec un verbe qui décrit une éventualité dont la culmination coïncide avec le point de perspective marqué par cet adverbe.

[18] La grande confusione in cui aveva sempre lasciato tutti i suoi libri, sparsi o ammucchiati qua e là sulle seggiole, per terra, sui tavolini, negli scaffali, lo fece ora disperare.

(CORIS : L. Pirandello, La mosca [1923], Milan, A. Mondadori, 1985 )

La grande confusion dans laquelle il avait toujours laissé tous ses livres, éparpillés ou entassés çà et là sur les chaises, par terre, sur les tables et les étagères, le fit ORA désespérer.

[19] Non avendo potuto una deputazione del suo esercito recatasi a Roma ottenere l'accoglimento delle sue richieste, Ottaviano giunse egli stesso a principio dell'agosto 43 alla capitale con il suo esercito e impose, con le buone e con le cattive, tutto. Egli si fece ora eleggere console e il I9 agosto 43 ebbe il consolato.

(Texte disponible sous: http://cronologia.leonardo.it/umanita/capo4I.htm)

Comme une délégation de l'armée qui s'était rendue à Rome n'avait pu obtenir l'accueil de ses demandes, Octavien arriva lui-même à la capitale au début du mois d'août 43 avec son armée et il imposa tout ce qu'il voulait. Il se fit ORA élire consul et le 19 août 43 il obtint le consulat. que le locuteur met laccent sur la victoire d’ Octavien. Il s'agit donc d'un mécanisme 
comparable à celui observé dans le cas de maintenant: ora, en tant qu'élément anaphorique, choisit comme point de repère le TR établi par l'événement qui a été décrit par le prédicat précédent au passé simple (l'arrivée d'Octavien à Rome), et il réoriente en même temps la perspective vers un événement postérieur, notamment l'élection d'Octavien comme consul ${ }^{8}$.

Pour résumer les observations faites dans cette dernière partie, on peut avancer l'hypothèse selon laquelle le trait caractéristique qui sépare maintenant français de ora italien est l'aboutissement d'un processus par lequel les possibilités de combinaison, et donc les restrictions vis-à-vis des propriétés aspectuelles, sont devenues progressivement plus conventionnelles. Cette évolution, qui devrait être confirmée par une étude de corpus plus systématique, se manifesterait par la restriction combinatoire avec l'aspect du prédicat. Si l'aspect perfectif n'est pas totalement exclu avec maintenant, il est restreint à des cas très spécialisés tels que les verbes phasiques et psychologiques.

Cette hypothèse, que nous proposons au demeurant à titre purement spéculatif, peut néanmoins avoir une répercussion sur l'analyse de ces adverbes. Nous verrons dans la deuxième partie de notre contribution, à la section 3, que les différences au niveau combinatoire sur le plan temporel se dédoublent en effet au niveau épistémique-argumentatif: c'est encore une fois ora qui admet un éventail de lectures plus varié que son pendant français or, qui s'est spécialisé pour exprimer des emplois plus centraux sur le plan argumentatif.

\subsubsection{Ora / maintenant dans le RN et le DIL}

La distinction formelle entre TR et PP a été introduite dans le système formel de la DRT pour rendre compte du fait que le TR, dans le système originaire de Reichenbach, revêt deux rôles distincts dans la construction dynamique du récit (Kamp, 2013). D’un côté, le TR introduit un repère temporel qui sert de point d'ancrage pour la progression du récit. De l'autre côté, il introduit un point qui sert de repère pour apprécier la perspective adoptée vis-à-vis des événements présentés dans le récit, c'est-à-dire le repère à partir duquel ces événements sont observés par le narrateur ou le locuteur. Ces deux emplois sont bien évidemment très différents, ce qui a amené Kamp (2013) (mais voir aussi Kamp et Rohrer, 1983) à y associer deux repères temporels distincts, en introduisant dans le système, à côté du TR, le PP ${ }^{9}$. Dans l'analyse de Kamp et Rohrer (1983), le dédoublement du TR en deux points de repère est motivé aussi, et précisément, par la sémantique

8. Nous remercions l'un de nos relecteurs pour avoir attiré notre attention sur le fait que, dans l'exemple [I9], l'adverbe ORA pourrait être traduit en français par alors. À propos de cet adverbe, voir entre autres Bras et al. (2006) et Le Draoulec et Bras (2007).

9. Ainsi, Kamp et Rohrer (1983:268) notent que: «some of the distinctions between some of these four categories [of anaphoric and indexical temporal adverbs] involve the notion of the temporal perspective. Modes of verbal presentation may differ in respect of the perspective from which they present the information they provide. The perspective may be either from the point of speech or from a temporal reference point that has been established by antecedent discourse». 
d'adverbes déictiques comme maintenant ${ }^{10}$. Dans ce qui suit, nous allons proposer une caractérisation sémantique de maintenant/ora à la lumière de cette hypothèse. Nous commencerons par un exemple attesté, et nous construirons des exemples comparables dans lesquels les variables contextuelles seront manipulées afin de mieux faire ressortir la contribution de maintenant dans l'agencement du récit. Ensuite, nous considérerons plus spécifiquement le contexte du DIL.

Considérons tout d'abord, en guise d'exemple, la phrase suivante en français (exemple $[20]$ ).

[20] Ma réponse appela une seconde lettre, où Moselly me fixait un rendez-vous à Paris. Il enseignait maintenant à Janson-de-Sailly. Il n'avait pas changé.

(Frantext: M. Genevoix, Trente mille jours, Paris, Seuil, 1980, p. 222)

La première phrase du texte reproduit sous [20] décrit l'enchaînement de trois événements. Le verbe au passé simple introduit l'événement correspondant à la réception d'une lettre par le narrateur. Cet événement fait suite à deux événements présupposés, qui correspondent à la réception d'une première lettre et à l'envoi d'une réponse à celle-ci. Le temps du passé ordonne ces événements par rapport à l'acte de parole du narrateur (EI). La séquence narrative peut donc être représentée comme en figure $\mathrm{I}$.

$\mathrm{La}$ réception de la deuxième lettre introduit le TR à ce stade du récit. La deuxième phrase du texte décrit la situation de Moselly, à savoir son état d'enseignant au lycée Janson-de-Sailly. On peut représenter aussi cet état comme une éventualité qui est localisée par le temps imparfait, et qui chevauche le TR introduit par la phrase précédente. Cette configuration est représentée en figure 2: au moment pertinent (celui de la réception de la deuxième lettre), Moselly enseigne à Janson-de-Sailly. Lettre 1 E4 E3 Lettre2$$
\text { TR }
$$
(acte de parole)

Figure 1

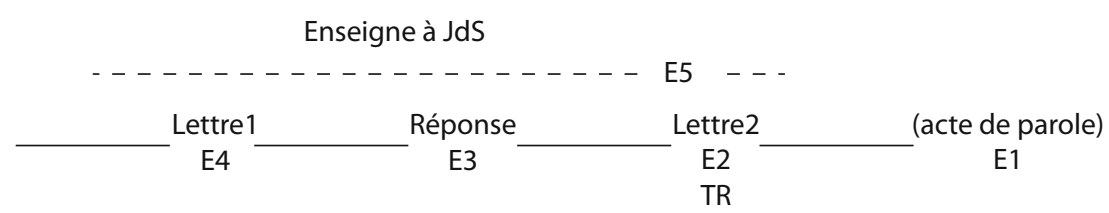

Figure 2

10. "If the author uses maintenant then the perspective shifts towards the reference point of the last sentence in the p.s. The author then tries to give the impression that the situation is viewed from that temporal angle [...]» (Kamp et Rohrer, 1983: 265). 
Considérons à présent la contribution de maintenant à l'intérieur de ce schéma narratif. Nous avons vu que maintenant, qui modifie la phrase exprimant $\mathrm{E}_{5}$, n'introduit pas un nouveau TR dans le récit. Si l'on manipule l'exemple [20] en faisant suivre la deuxième phrase par un prédicat qui introduit l'occurrence d'un nouvel événement, comme dans [2I], cet événement étant localisé par rapport au TR introduit par $\mathrm{E}_{2}$ : on comprend que le narrateur a appelé Jacques après avoir reçu la deuxième lettre. Ce nouvel événement fait d'ailleurs avancer le TR du récit, alors que la situation de Moselly continue à chevaucher ces repères temporels.

[21] Ma réponse appela une seconde lettre, où Moselly me fixait un rendez-vous à Paris. Il enseignait maintenant à Janson-de-Sailly. J'appelai mon ami Jacques.

En revenant à notre exemple attesté, considérons la continuation de [20]. La troisième phrase, «Il n'avait pas changé», décrit un état, exprimé au plus-que-parfait, qui devrait se superposer à celui d'enseigner à Janson-de-Sailly et, de ce fait, aux trois événements qui s'enchaînent dans le récit. Cependant, cette phrase introduit aussi, à cause du sens même du prédicat changer, une présupposition. On ne peut comprendre cette continuation à moins d'évoquer une situation ou un état par rapport auxquels Moselly aurait pu changer. Cette évocation se situe en dehors des faits objectifs rapportés dans le texte et appartient plutôt au plan subjectif du narrateur. C'est précisément vis-à-vis de cette évocation qu'on peut apprécier la contribution de maintenant.

Pour apprécier la contribution de ora et maintenant nous devons considérer un repère temporel qui n'appartient pas à l'ensemble des relations qui localisent les événements sur l'axe temporel et qui décrivent l'enchaînement des faits comme si le récit était une représentation de la réalité. Comme le suggèrent déjà Kamp et Rohrer (1983), il est plutôt nécessaire de prendre en considération les relations temporelles dans le contexte fictif que le récit dessine. On introduira ainsi la perspective des acteurs du récit. Ce qui se passe en [20] avec l'introduction de maintenant est justement un changement du PP: ce PP n'est plus indexé au moment d'énonciation correspondant à l'événement de l'acte de parole EI, mais devient interne au récit. Le PP adopté dans ce cas est indexé au dernier TR, qui évalue la situation de Moselly au moment de la réception de la lettre.

Nous pouvons représenter ce mouvement du PP en supposant qu'il est ancré à un acte de perception interne au récit (figure 3), qui correspond à une révélation due au contenu de la deuxième lettre.

L'état d'enseigner à Janson-de-Sailly est donc anaphorique par rapport au TR du récit (d'où l'utilisation de l'imparfait) mais, grâce à la contribution sémantique de maintenant, il est introduit comme coïncidant avec un acte de perception qui marque la prise en charge d'un nouveau PP au moment correspondant au repère TR de la part du narrateur. Cette éventualité est actualisée par rapport au PP introduit à ce stade du récit. Finalement, c'est le changement du PP qui crée les conditions pour la continuation de [20], à savoir l'évocation d'un état précédent, différent de celui exprimé par E5, qui aurait pu justifier un changement de Moselly. 
Récit

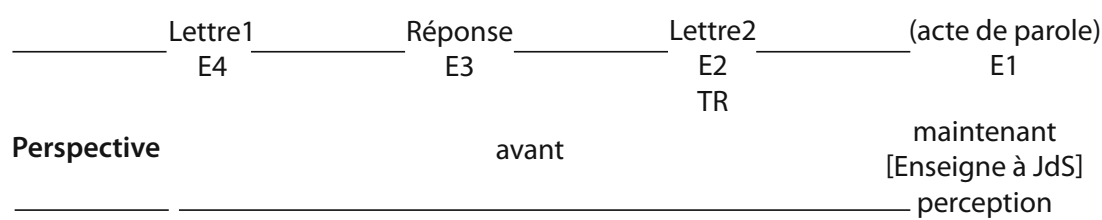

Figure 3

L'exemple que nous avons discuté nous montre que le changement de la perspective interne au récit peut être associé à l'introduction d'un nouveau repère temporel. Dans l'exemple proposé, nous avons fait correspondre ce nouveau repère à un événement distinct (un acte de perception). L'introduction d'un acte de prise en charge interne au récit nous permet de garder le principe général du rapport anaphorique entre le $\mathrm{PP}$ et le TR plus saillant dans le contexte à travers les différents modes de discours.

L'analyse de maintenant/ora que nous avons proposée pour le RN, peut être étendue facilement aux contextes de DIL, où le TR et le PP appartiennent à deux niveaux de représentation qui sont par définition distincts. Il est généralement admis que dans le DIL, on trouve d'un côté la représentation des faits «objectifs» de la part du narrateur (la présentation des faits comme s'il s'agissait de l'ordre du temps dans le monde réel) et, de l'autre, la représentation «subjective» de ces mêmes faits, représentation qui suit la perspective des personnages du récit (et qui, de ce fait, peut revêtir un caractère polyphonique ${ }^{11}$ ). Dans ce contexte aussi, maintenant/ora agissent comme des adverbes anaphoriques par rapport à un TR objectif. La fonction d'actualisation, en revanche, se manifeste par rapport à un PP qui se situe au niveau subjectif de la représentation, dont l'introduction doit être accompagnée par un acte, implicite ou explicite, de «prise en charge» de la part d'un personnage interne au récit. Nous pouvons donc proposer, dans ce cas aussi, une contrainte formelle qui régit l'utilisation de maintenant/ora: la présence d'un acte de prise en charge de la perspective par un locuteur interne. Avec Guéron (20I5), nous proposons de représenter cet acte de prise en charge comme un événement implicite, qui appartient à l'ordre temporel sur le plan de la perspective.

Pour conclure, nous pouvons reprendre la question soulevée au début: quel est l'effet de ora sur le récit? La réponse est que le PP, qui devrait être calculé par défaut vis-à-vis de l'acte d'énonciation, est mis à jour en étant indexé au dernier TR du récit, comme le suggèrent Kamp et Rohrer (1983). C'est donc par rapport au temps de perspective (TP), qui est un TR subjectif calculé par rapport à l'événement de prise en charge, que ora déploie son potentiel de mise à jour.

11. Voir Ducrot (I980 et 1984), qui emprunte ce terme à la théorie littéraire, en particulier aux travaux de Bakhtin. 
Considérons encore une fois l'exemple attesté [9], repris ici sous [22].

[22] [Et je me gardais bien de dire à Marie: je vois la mort, je vois la mort, elle est dans cette maison, en train de ramasser l'enfançon!] Parce que Marie était en grande colère, elle n'avait plus son regard de lavande mouillée. Maintenant, elle jetait des yeux furibonds de côté et plumait dans son tablier les cailles avec férocité, avec méchanceté, toute pâle, énervée, échevelée.

(Frantext: A.-M. Garat, Les mal famées, Arles, Actes Sud, 2000, p. 149)

Le PP du récit, tel qu'il est présenté dans la première phrase du passage reproduit sous [22], est conçu comme étant postérieur aux événements décrits dans le texte précédent (reproduit entre crochets), qui sont aussi exprimés au passé. Maintenant a pour effet d'actualiser le PP en l'ancrant au TR introduit par un changement d'état, tel qu'il est présupposé par l'utilisation de la négation ne... plus dans l'expression «elle n'avait plus son regard de lavande mouillée». Pour terminer, examinons aussi un exemple de récit attesté en italien (exemple [23]).

[23] L'atto legale, che stava preparando l'avvocato, le piaceva molto e l'appagava. Abituata sempre ad obbedire e poi trattata peggio d'una serva dal marito, ora anche lei aveva un avvocato che pensava ai suoi interessi; ed era impazientissima che l'atto fosse completato.

(CORIS : F. Tozzi, Il podere [192I], Milan, Rizzoli, I983)

L'acte légal que l'avocat était en train de préparer lui plaisait beaucoup et la comblait. Habituée comme elle l'avait toujours été à obéir et puis traitée pire qu'une esclave par son mari, ORA elle aussi avait un avocat qui s'occupait de ses intérêts; et elle attendait avec impatience que cet acte fût terminé.

Dans l'extrait de récit représenté dans l'exemple [23], ora actualise le PP en l'ancrant à un changement qui ne concerne pas tant les événements majeurs qui s'enchaînent dans le récit que l'état psychologique du personnage principal. Remarquons en effet que la rédaction de l'acte est toujours en cours, comme le signale la périphrase progressive dans la relative enchâssée (stava preparando). La phrase suivante dans le récit insiste sur le fait que l'acte juridique n'est pas encore terminé au moment où le nouveau PP est fixé.

\subsection{Conclusions}

Dans notre analyse de l'emploi temporel de maintenant/ora, ces adverbes sont traités comme des opérateurs ayant la fonction d'introduire un indice temporel qui est mis en relation avec un repère saillant dans le contexte. Nous proposons, en guise de conclusion pour cette section, une représentation formelle simplifiée de la contribution temporelle de maintenant/ora, qui n'inclut pas les restrictions plus spécifiques que nous avons discutées dans la section 2.2.I. Nous pouvons représenter la sémantique de maintenant/ora comme une fonction $f$ qui introduit une variable $\left(t_{m}\right)$ dans son domaine d'application. La variable $t_{m}$ est sensible au contexte, en ce sens 
que la fonction assigne à cette variable la valeur du repère temporel $t_{c}$ déterminé par le contexte dans lequel maintenant/ora apparaît. Le contenu propositionnel de la phrase hôte $Q$ est ensuite évalué vis-à-vis de ce repère, comme l'indique le deuxième conjoint de [24].

[24] soit $\mathrm{Q}\left(\mathrm{t}_{\mathrm{c}}\right)$ :

maintenant/ora $(\mathrm{Q})=f\left(\mathrm{t}_{c}, \mathrm{t}_{\mathrm{m}}\right) \& \mathrm{Q}\left(\mathrm{t}_{\mathrm{m}}\right)$

Suivant cette définition, la valeur de maintenant/ora à travers les différents modes du discours dépend de la possibilité d'assigner à $t_{m}$ un indice contextuel donné. Le DD, illustré par [4], correspond au contexte dans lequel maintenant/ora déploient leur fonction de déictiques "purs». L'indice temporel $t_{m}$ associé avec maintenant/ora est mis en relation avec le temps d'occurrence de l'acte d'énonciation $e_{I}$ de la proposition $Q$ (voir l'exemple [25]). L'indice contextuel qui donne la valeur à $t_{m}$ est donc le temps $t_{t}$ correspondant à l'acte d'énonciation, et c'est vis-à-vis de ce repère qu'est ensuite évaluée la vérité de $Q$.

[25] a. $\mathrm{e}_{1}\left(\mathrm{Q}, \mathrm{t}_{1}\right) \& \mathrm{Q}:$ Il fait beau

b. maintenant $(\mathrm{Q})=f\left[\mathrm{t}_{1}, \mathrm{t}_{\mathrm{m}}\right] \& \mathrm{Q}\left(\mathrm{t}_{\mathrm{m}}\right)$

[où $\mathrm{t}_{\mathrm{m}}$ : indice temporel associé à maintenant; $\mathrm{t}_{\mathrm{l}}$ : indice temporel associé à $\mathrm{e}_{\mathrm{t}}$ ]

Dans cette section, nous avons aussi proposé une analyse de la contribution de ora/maintenant dans le RN et dans le DIL, en les décrivant comme des adverbes qui mettent en relation l'indice temporel $t_{m}$ avec le repère temporel correspondant au PP interne au récit. Nous avons vu que le PP doit être dissocié du TR introduit par les événements décrits dans le contexte précédent. Considérons à nouveau l'exemple [22]. Le TR de la phrase où apparait maintenant est ordonné par rapport à un événement $e_{t}$ qui appartient à l'ordre des événements présupposés, et qui décrit un changement d'état. Dans le segment narratif reproduit en [22], la présupposition du changement d'état (Marie avait son regard [...] $\rightarrow$ Marie n'avait pas son regard) est véhiculée par l'élément négatif plus. Dans le premier conjoint de [26a], la présupposition est représentée entre crochets, alors que le deuxième conjoint représente l'assertion de l'état résultant $Q 2$, qui correspond à la première phrase de [22]. Le TR de $Q 2$, qui coïncide avec $t_{2}$, est donc successif à $e_{I} / t_{1}$. Considérons à présent la phrase successive du segment [22], que nous appellerons $Q_{3}$. Le prédicat de $Q_{3}$ est modifié par l'aspect imperfectif, dont la spécificité, comme nous l'avons vu, est de ne pas introduire un nouveau TR qui ferait progresser le récit. L'indice temporel $t_{3}$ introduit par $Q_{3}$ n'est donc pas ordonné vis-à-vis du TR précédent, qui est toujours indexé à $t_{2}$ (exemple [26c]).

[26] [Parce que Marie était en grande colère,] elle n'avait plus son regard de lavande mouillée. Maintenant, elle jetait des yeux furibonds de côté.

a. $\left[\right.$ Pres: $\left.\mathrm{e}_{1}\left(\mathrm{Q}_{1}, \mathrm{t}_{1}\right)\right] \& \mathrm{Q}_{2}\left(\mathrm{t}_{2} / \mathrm{TR}\right)$ : Marie n'avait plus son regard de lavande mouillée.

b. $\mathrm{e}_{3}\left(\mathrm{Q}_{3}, \mathrm{t}_{3}\right) \& \mathrm{Q}_{3}:$ Marie jetait des yeux furibonds de côté.

c. $\mathrm{t}_{1}<\left\{\mathrm{t}_{2} / \mathrm{TR}, \mathrm{t}_{3}\right\}$ 
Dans ce contexte, quel est donc l'apport de maintenant? Nous avons vu que maintenant n'introduit pas un nouveau TR pour sa phrase hôte. La contribution de cet adverbe se joue sur le plan du TP, qui est caractéristique du mode narratif. Sa fonction est celle de mettre en relation l'indice temporel qu'il introduit $\left(t_{m}\right)$ avec celui du TP, que nous représentons par la variable $t_{p}$ dans [26d].

\section{[26] d. maintenant $\left(\mathrm{Q}_{3}\right)=f\left[\mathrm{t}_{\mathrm{p}}, \mathrm{t}_{\mathrm{m}}\right] \& \mathrm{Q}_{2}\left(\mathrm{t}_{\mathrm{m}}\right)$}

Du point de vue des relations temporelles dans le récit, le rapport entre $t_{3}$ et $t_{2} / T R$ n'a pas changé, car $e_{3}$ n'a pas fait avancer le TR de la narration. Cependant, maintenant a introduit une relation explicite entre l'indice temporel de cet événement et le TP du récit. La proposition $Q_{3}$ doit donc être évaluée par rapport à $t_{2} / T R$ pour ce qui est de sa valeur de vérité sur le plan des faits présentés par la narration, et par rapport à $t_{p}$ vis-à-vis de la cohérence interne au récit, telle qu'elle est donnée par la perspective adoptée dans la présentation de ces mêmes faits.

Dans le contexte du RN et du DIL, et seulement dans ces contextes, la fonction de ora/maintenant, qu'on déduit de leur contenu lexical d'adverbes déictiques, est celle d'actualiser explicitement la perspective. Comme le montrent les exemples que nous avons analysés jusqu'ici, et comme il apparaît particulièrement dans le dernier exemple sous [22], dans les contextes narratifs, maintenant/ora ont un contenu spécialisé, qui est celui de mettre en relief un PP qui sépare deux états dans un rapport d'asymétrie en termes de saillance. Les adverbes maintenant/ora séparent d'une façon tranchante un nouvel état par rapport à un état antérieur qui passe à l'arrière-plan. Qui plus est, la perspective du locuteur change, passant de l'orientation vers ce qui a été auparavant (le passé, l'arrière) à l'orientation vers l'avant, pour mettre en relief un nouvel état qui se déploie au moment de la saisie. Nous verrons dans la section suivante que ce même noyau sémantique est à la base de la spécialisation des deux adverbes comme marqueurs discursifs.

\section{Perspective argumentative}

Dans cette deuxième partie, nous étudierons plus en détail la fonction argumentative de l'expression française or et de l'italien ora, toujours dans une perspective comparative. En particulier, nous allons dégager deux aspects qui nous semblent primordiaux pour la caractérisation de la fonction argumentative de or et ora:

I. d'un côté, nous essaierons de déterminer si le signifié de or contient la notion d'adversativité (voir Métrich et al., $1993^{12}$; et Fernández, 1998) ou si l'expression de cette notion relèverait plutôt d'une lecture pragmatique réservée à certains contextes;

12. Métrich et al. (1993: II5), dans le dictionnaire sur Les invariables difficiles, rangent or parmi les équivalents français du connecteur adversatif allemand aber et juxtaposent en tant qu'expressions proches ou quasi synonymes mais, cependant, pourtant et toutefois (voir aussi p. II8 où or apparaît conjointement avec mais, au contraire et contrairement à). 
2. de l'autre côté, nous examinerons le rapport entre les lectures temporelles et argumentatives de ora. Existe-il des traits sémantiques communs aux deux lectures? Est-il possible de postuler une caractérisation sémantique abstraite pour cet adverbe. Plus précisément, nous proposons de répondre à la question suivante: peut-on défendre l'hypothèse selon laquelle la lecture argumentative se fonde sur la lecture temporelle?

\subsection{Relation adversative de or}

En ce qui concerne la question exprimée sous le point $(\mathrm{I})$, le dépouillement des exemples tirés de corpus journalistiques ${ }^{13}$ révèle que le marqueur discursif or est compatible avec un ensemble de lectures très varié. Cela indique tout d'abord que or n'inclut pas le trait adversatif dans son noyau sémantique. Au contraire, dans un grand nombre de contextes - comme nous le verrons dans ce qui suit -, la fonction de or se limite à réorienter l'argumentation. En même temps, une analyse plus raffinée des données laisse entrevoir des degrés différents d'orientation oppositive dans le rapport entre les deux segments discursifs reliés par ce marqueur. Il est donc possible d'ordonner les différentes lectures établies par or sur une échelle qui va d'un simple rapport de réorientation de l'argumentation à des relations nettement antithétiques, clairement adversatives.

Regardons de plus près les différents types de rapport qu'on peut resituer sur l'échelle des rapports oppositifs entre les deux segments du discours concernés. Nous commençons par des contextes dans lesquels or évoque un rapport qu'on peut caractériser comme, tout au plus, faiblement oppositif. En effet, dans les exemples comme [27], or ne fait qu'introduire un nouvel argument, orienté de manière à relancer l'argumentation et à la faire avancer dans un sens déterminé.

[27] Dans nombre d'accidents étudiés, aucun dépistage n'a pu être réalisé. Le plus souvent parce que les victimes se trouvaient dans un état déplorable. «Or plus c'est grave, plus l'alcool est impliqué», analyse le médecin.

$$
\text { (Le Monde, 26/II/2002, p. II) }
$$

Dans l'exemple [27], or introduit une proposition (plus $x$, plus $y$ ) qui explique et spécifie de ce fait la proposition antérieure ayant trait à l'état déplorable des victimes. Un effet de contraste plus fort est produit par l'introduction d'un argument, dont le caractère est signalé par le marqueur or comme étant inattendu, surprenant ou même inouï. Dans ces contextes de contraste plutôt faible, l'information saillante, et dans certains cas même spectaculaire, s'inscrit dans un cadre argumentatif établi, c'est-à-dire qu'elle reste dans le même frame (ou cadre thématique) tout en ouvrant une nouvelle boucle dans le déroulement de l'argumentation. Dans l'exemple [28], l'argument introduit par or s'inscrit dans le cadre de l'exploitation pétrolière, mais ce marqueur discursif souligne en même temps un nouveau fait qui est présenté comme étant ignoré par le lecteur et donc susceptible de produire un effet d'étonnement.

13. Pour la constitution du corpus, voir note I. 
[28] Mais la plus grande révolution reste certainement à venir. Actuellement, lors de l'exploitation d'un gisement pétrolier, seuls $35 \%$ des hydrocarbures présents sont extraits. «Or, la récupération de i \% des $65 \%$ restants représente trois ans de consommation pétrolière mondiale», précise Bernard Montaron.

(Le Monde, 22/II/2002, p. 27)

Dans un grand nombre de contextes, l'effet contrastif est majeur, car ce que le fait nouveau introduit dans le discours est à même de contrecarrer une attente fortement enracinée. Or marque donc une tournure imprévue dans l'acheminement de l'argumentation. Dans l'exemple [29], l'argument introduit par or est destiné à renverser la direction de l'argumentation.

[29] Marie-France l'explique: «Je ne vois pas comment ce mouvement, qui est haï par la population et qui est obligé de s'imposer par les armes, tout en obéissant au Rwanda, pourrait être accepté comme le gouvernement légitime. Or, tournant décisif d'une guerre où les fronts se sont stabilisés depuis deux ans, le Rwanda s'est soudainement retiré de RDC deux semaines plus tôt».

(Le Monde, 07/II/2002, p. 4)

Si dans le segment antérieur, qu'on peut appeler $A$, le lecteur est porté à croire que la situation conflictuelle est caractérisée par la stabilité des fronts ainsi que par un problème de légitimité de la part du régime vainqueur, le segment $B$ suivant, introduit par or, renverse le tableau des rapports de force : le fait présupposé (stabilité des fronts) est remis en question par un nouveau fait (retraite de RDC, les vainqueurs perdent leur soutien), ce qui entraîne de nouvelles conséquences possibles (les vainqueurs perdent leur position, le conflit de légitimation peut s'aggraver).

Dans quelques contextes, la proposition introduite par or suggère une conclusion qui exclut une des propositions introduites dans le segment antérieur. Parallèlement, l'inférence selon laquelle l'alternative nouvellement introduite est la seule valable s'impose. Autrement dit: si $q_{I}$ et $q 2$ forment une alternative théorique, mais que $q I$ est une proposition fausse dans le monde réel, alors il s'ensuit que $q 2$ est la seule proposition possible (ou vraie). Du coup, l'emploi de or rectifie un raisonnement en invalidant l'hypothèse qI. C'est ce schéma que nous retrouvons dans l'extrait sous [30].

[30] «Quand une banque gagne $0,5 \%$ de parts de marché en France, c'est énorme! relève Se Ting Tan. Il y a deux solutions pour gagner des parts de marché: gagner de nouveaux clients ou vendre davantage de produits. Or les Français sont très fidèles à leurs banques, le taux de fidélité dépassant $95 \%$, l'un des plus élevés en Europe.» (Le Monde, 08/10/2002, p. I8)

Dans des contextes d'opposition encore plus marqués, la proposition où apparât l'expression or présente un argument qui va à l'encontre d'un état de choses (par exemple une attitude, une attente, une intention, une tentative qui vise à la réalisation d'une situation). Dans ces contextes, les faits décrits dans la proposition introduite par or entravent la réalisation d'une situation envisagée dans le segment antérieur. 
Dans l'exemple [31], la proposition $p$ («attendre des mesures précises [...] [pour] faire de l'éducation une priorité») implique la conséquence $c$ (embaucher plus de surveillants et d'aides-éducateurs). Cependant, l'état des choses décrit par $q$ ( «le budget demeure, avec ses suppressions de postes») va à l'encontre de ce qui devrait être la conséquence $c$ et implique plutôt $c^{\prime}$ (il y aura encore moins de surveillants et d'aides-éducateurs).

[3I] Il reste évasif quant au nombre de surveillants et d'aides-éducateurs disponibles dans les établissements scolaires à la rentrée 2003. Georges Dupon-Lahitte, président de la FCPE, principale fédération des parents d'élèves, attend «des mesures précises traduisant véritablement la volonté du gouvernement de faire de l'éducation une priorité». «Or le budget demeure, avec ses suppressions de postes et l'abandon du plan pluriannuel de recrutement décidé par le gouvernement Jospin», constate M. Dupon-Lahitte.

(Le Monde, 09/12/2002, p. 7)

Dans l'exemple [32], le fait exprimé dans la deuxième proposition ( $p$ : ces boîtes sont toujours commercialisées) contredit fondamentalement une demande rapportée dans le segment antérieur, qui vise à la réalisation de non-p.

[32] Ainsi, pour certains jouets «fonctionnels» comportant des outils miniatures, répliques de modèles adultes (comme les scies dans des coffrets de bricolage, ou les minicutters dans une mallette d'éveil artistique) et ayant provoqué plusieurs blessures, la CSC avait demandé leur retrait du marché du jouet et leur remplacement par des objets en matière plastique. «Or ces boîtes, généralement importées d'Asie, sont toujours commercialisées», constate Patrick Mesnard, conseiller technique à la CSC.

(Le Monde, 20/II/2002, P. 32)

Dans la version la plus forte du rapport contrastif entre le segment antérieur et le segment introduit par le marqueur or, ce dernier segment est en antithèse avec l'état des choses décrit précédemment. Dans l'exemple [33], la proposition p2 introduite par or ("quand un élève a 5 , il vaut 5 ») exemplifie, à travers la mention d'un cas spécifique, le fait que l'état des choses caractérisé précédemment dans la proposition $p_{I}$ («la note n'a rien à voir avec la valeur de la personne») ne se vérifie pas dans le monde réel. La proposition $p 2$ avance donc une instance qui réfute la validité de la maxime générale invoquée dans le segment précédent.

[33] Il m'a dit: «La note n'a rien à voir avec la valeur de la personne. Or, dans l'institution, quand un élève a 5 , il vaut $5 »$. Pendant ces deux ans de BEP vente, Cyril est docile. (Le Monde, $14 / \mathrm{II} / 2002$, p. $\mathrm{I} 2$ )

Le cas présenté dans l'exemple [34] est tout à fait comparable. La proposition $p 2$ («sans nul doute la fin de la guerre profitera aux deux rois») constitue l'antithèse de la proposition $p_{I}$ («Qui a le profit de la guerre, il en a le vrai honneur»). Cette proposition $p 2$ est étayée par un argument qui revêt le caractère d'une maxime ( «le vrai honneur et pleine victoire de la guerre, c'est la vraie paix»). 
[34] Mais Philippe leur répond par le proverbe commun : «Qui a le profit de la guerre, il en a le vrai honneur». Or sans nul doute la fin de la guerre profitera aux deux rois: le vrai honneur et pleine victoire de la guerre, c'est la vraie paix.

(Le Monde, 23/10/2002, p. 19)

Les exemples que nous venons d'analyser partagent un certain nombre de caractéristiques permettant de faire un résumé provisoire. Nous pouvons donc proposer les conclusions suivantes:

I. or marque la mise à jour (update) de l'argumentation par le biais d'un enrichissement du fonds commun;

2. il confère un statut proéminent à la proposition ajoutée au fonds commun: dans le contexte or $q$, la proposition $q$ en tant qu'argument le plus récent, acquiert le statut de l'argument le plus saillant et pertinent dans le cadre d'un raisonnement étalé; étant donné son caractère proéminent dans la hiérarchie des arguments, or actualise et réoriente le déroulement et la direction du raisonnement. Reprenons, par exemple, le contexte exposé en [3I] : l'attente de la part du président de la FCPE «des mesures précises traduisant $[. .$.$] la volonté [...] de faire de l'éducation une priorité»,$ véhiculée par la proposition $p$, est un argument pour la conséquence $c$ (le gouvernement doit prendre des mesures politiques, en particulier, embaucher des aides-éducateurs, en vue d'atteindre l'état des choses décrit par $p$ ). Cependant, l'état des choses décrit par $q$ («le budget demeure, avec ses suppressions de postes [...]») s'oppose à $c$ et aboutit à la conséquence $c$ ' (le gouvernement ne fait pas de l'éducation une priorité et il y aura encore moins d'aides-éducateurs). La proposition $q$ ("Or le budget demeure, avec ses suppressions de postes [...]») exprime l'argument le plus proéminent, en ce qu'elle invalide l'argument véhiculé par $p$ (l'attente du président de la FCPE en faveur de la priorité de l'éducation) et réoriente, de cette manière, le déroulement de l'argumentation (étant donné q, l'éducation n'est pas une priorité du gouvernement);

3. la mise en relief de la proéminence d'un nouvel argument et la fonction de réorganisation reliée à ce caractère proéminent se trouvent, en plus, à la base d'effets de contraste dont le degré sur une échelle donnée varie selon les contextes;

4. la gamme de rapports entre le segment antérieur et la proposition introduite par or s'étend d'une simple réorientation donnée à l'argumentation à une forte antithèse à l'état de l'argumentation atteint à un moment donné, passant par des degrés intermédiaires comme, par exemple, les tournures inattendues, les attentes contrecarrées ainsi que l'annulation de solutions envisagées. Nous avons pu apprécier cette gamme de rapports oppositifs dans les exemples [27] à [34]. Si [27] est un exemple illustrant un simple contraste thématique - d'un côté la perspective des victimes, de l'autre le rôle joué par 
l'alcool -, [34] représente le cas des relations nettement antithétiques entre deux propositions qui expriment des maximes contraires.

Force est de constater que la fréquence de l'emploi de or dans des contextes qui accentuent l'opposition entre le segment antérieur et la proposition qu'il introduit, renforce l'inférence pragmatique (implicature) de contraste associée à ce marqueur discursif; il n'en reste pas moins vrai, cependant, que l'implicature de contraste liée à or ne représente qu'une implicature conversationnelle, dépendante du contexte créé par le locuteur. Finalement, il n'est pas improbable que cette implicature d'opposition puisse acquérir un statut conventionnel, au fur et à mesure que les locuteurs généralisent les emplois de or dans des contextes clairement adversatifs, c'est-à-dire dans des contextes qui opposent deux arguments appartenant au même niveau argumentatif de manière à créer un rapport clairement antithétique entre le segment antérieur et la proposition introduite par or.

\subsection{Lecture temporelle et argumentative de ora}

Passons maintenant à l'équivalent italien de or et attardons-nous sur le marqueur ora. Rappelons tout d'abord que ora est utilisé tant comme adverbe temporel (voir la section 2) que comme marqueur discursif avec une valeur argumentative. Ses emplois recouvrent tout le champ fonctionnel qui est partagé par maintenant (temporel) et or (argumentatif) dans la langue française.

Si l'on passe en revue les différents contextes où ora est utilisé comme marqueur discursif, on s'aperçoit que dans cet emploi, ora recouvre également une gamme assez large de rapports d'opposition. On repère, par exemple, au pôle des rapports d'opposition faible, des exemples dans lesquels ora ne fait que réorienter l'argumentation, comme en [35].

[35] Tosca, a Trieste, non si dava dal 1978, quando Alberto Fassini la mise in scena con Raina Kabaivanska. Ed è questo allestimento che è stato scelto per l'inaugurazione di quest'anno, ma con interpreti tutti nuovi. [...] Ora, inaugurare una stagione con un vecchio allestimento è un atto di coraggio, che toglie clamore alla serata e concentra le attese sugli esiti musicali dello spettacolo. Tuttavia la scelta di questa Tosca di Fassini non ci sembra una scelta felice.

(D. Villatico, La Repubblica, 1985)

Tosca, à Trieste, n'avait pas été représentée depuis 1978, quand Alberto Fassini la mit en scène avec Raina Kabaivanska. Et c'est justement cette mise en scène qui a été choisie pour l'inauguration de cette année, avec des interprètes nouveaux. [...] ORA, inaugurer la saison avec une ancienne mise en scène est un acte de courage, qui étouffe le bruit autour de la soirée et concentre les attentes sur la performance musicale du spectacle. Cependant, le choix de cette Tosca de Fassini ne nous paraît pas heureux.

Dans le passage reproduit sous [35], ora met en relief une information déjà avancée dans le segment antérieur, mais qui est reprise comme point de démarrage choisi pour donner une nouvelle tournure à l'argumentation. En d'autres termes, 
ora marque le fait reformulé («inaugurer la saison avec une ancienne mise en scène») comme étant un élément saillant qui permet la transition avec le segment suivant de l'argumentation. Il est particulièrement intéressant de voir que la tournure adversative que prend l'argumentation ne se manifeste à part entière qu'au moment où le marqueur tuttavia («cependant») est mis au premier plan. Ce n'est qu'à partir de ce moment-là que le mouvement anti-orienté du discours se déploie pleinement ${ }^{14}$. Le rapport entre le segment antérieur et la proposition qui contient ora est plus accentué dans l'exemple [36].

[36] A cominciare proprio dalla dizione del tedesco, che faceva la stessa impressione che fa a un italiano l'italiano in bocca britannica. I cantanti (tranne il basso Cornelius Hauptmann, che dal nome sembra tedesco e andava un po' meglio) pronunciano tutti vocali indefinite e sorvolano sulle consonanti. Ora il tedesco è una lingua ricchissima di consonanti e proprio alle consonanti affida spesso la sua forza espressiva. Questa dizione manchevole risultava disastrosa nel tenore che faceva la parte dell'Evangelista.

(La Repubblica, 1989)

À commencer justement par la prononciation de l'allemand, qui faisait la même impression que ferait, à un italien, l'italien parlé par un anglais. Les chanteurs (sauf Cornelius Hauptmann, à voix de basse, dont le nom paraît allemand et qui s'en sortait un peu mieux) prononcent tous des voyelles non défnies et survolent sur les consonnes. ORA, l'allemand est une langue très riche en consonnes, et qui confie justement aux consonnes sa force expressive. Cette prononciation défectueuse avait un effet désastreux dans le cas du ténor dans le rôle de l'Evangelista.

Dans [36], ora introduit un fait (décrit par $p$ ) dont la conséquence est contraire à l'information avancée dans le segment antérieur. Du fait $p$ («l'allemand est une langue très riche en consonnes, et qui confie justement aux consonnes sa force expressive») découle la conséquence $c$ selon laquelle on aurait dû faire attention à l'articulation appropriée des consonnes. Cependant, cette conséquence va à l'encontre des informations fournies par le segment antérieur $q$ («les chanteurs [...] prononcent tous des voyelles non définies et survolent sur les consonnes»). La proposition introduite par ora avance un fait général d'ordre encyclopédique qui sert d'argument anti-orienté par rapport à la direction argumentative qu'on peut saisir dans le segment antérieur. Dans deux autres configurations le rapport d'opposition est beaucoup plus accentué. L'extrait sous [37] illustre un exemple très caractéristique. Dans le segment antérieur le locuteur affirme une sorte de maxime ou de principe général. La proposition introduite par ora, cependant, avance un cas spécifique qui contredit la maxime ou le principe invoqué(e).

[37] In una democrazia rappresentativa, i politici sono per definizione una «classe aperta», con uscite ed entrate frequentissime. [...] Ora, nessuno in buona fede può affermare

14. Pour la distinction entre énoncés co- et anti-orientés, voir Anscombre et Ducrot (1988). 
che i politici in Italia siano una classe aperta e che le entrate e le uscite, cioè il rinnovamento del personale, sia soddisfacente.

(E. Scalfari, La Repubblica, I986)

Dans une démocratie représentative, les politiciens sont, par définition, une "classe ouverte", avec des sorties et des entrées très fréquentes. [...] ORA, personne ne peut affirmer en toute bonne foi que les politiciens en Italie sont une classe ouverte, et que les entrées et sorties, c'est-à-dire le renouvellement du personnel, sont satisfaisantes.

La proposition personne ne peut affirmer que... constitue donc une sorte d'antithèse de la maxime établie dans le segment précédent et c'est ora qui exerce la fonction de marquer le passage à une réorientation de l'argumentation, dont la direction est contraire à ce qui a été présenté comme information pertinente dans le segment antérieur. En d'autres termes, on passe d'une réflexion générale portée sur un type de régime politique à la situation spécifique que l'on croit pouvoir identifier comme réelle pour l'Italie.

Discutons un dernier exemple, qui montre un rapport de contraste accentué entre le segment précédent et la proposition marquée par ora. Dans l'exemple [38], le locuteur joue sur des attentes ou bien sur des probabilités par défaut attribuées à des rapports établis (à la manière d'une implication) entre les faits en question.

[38] Tanto per cominciare, il Presidente Nordio ha detto a Minoli, che lo interpellava sulla sicurezza aerea quanto segue: le probabilità che un pilota ha di fare un gesto sbagliato (e qualche volta, fatale) sono le stesse probabilità che abbiamo noi di partire in macchina col freno tirato. Ora considerando che parto sempre in macchina col freno tirato, e sempre giurando di non farlo più, avevo deciso di rinunciare all' aereo e di raggiungere la città di Milano a piedi, o in bicicletta.

(B. Placido, La Repubblica, 1988)

Pour commencer, le président Nordio a dit à Minoli, qui le questionnait à propos de la sécurité aérienne, ce qui suit: les probabilités pour un pilote de faire un mauvais geste (et parfois un geste fatal) sont les mêmes que celles qu'on aurait de démarrer la voiture avec le frein à main tiré. ORA, étant donné que je démarre toujours avec le frein à main tiré, et toujours en jurant que je ne le ferai plus jamais, j’avais décidé de renoncer à l'avion et de me rendre à Milan à pied, ou à vélo.

En ce qui concerne le rapport établi entre le fait de faire un mauvais geste et celui de démarrer la voiture avec le frein à main tiré, on s'attendrait à une probabilité minimale. Le locuteur donne une tournure inattendue à son argumentation et invalide les attentes présupposées en mettant en avant ses propres expériences, qui sont censées annuler le rapport établi dans le segment antérieur (et, de ce fait, la direction de l'argumentation). Encore une fois, l'effet d'opposition trouve son origine dans le contraste entre un fait général et le cas spécifique qui est à même d'invalider ce fait général. Dans notre exemple, le locuteur oppose des attentes - partagées et, au contraire, individuelles - tout en jouant sur la quantification des probabilités 
attendues (une probabilité presque nulle $v$ s une probabilité maximale, rendue explicite par le quantificateur sempre/toujours). Pourtant, force est de constater que, dans les deux exemples présentés, les locuteurs exploitent le contraste entre le général et le particulier, ainsi qu'entre les attentes partagées et une instance inattendue ou exceptionnelle, sans qu'on puisse affirmer que les deux faits opposés sont au même niveau. Par conséquence, on ne peut décrire le rapport entre l'état des choses exposé dans le segment antérieur et la proposition introduite par ora en termes d'antithèse ou de réfutation directe.

Pour résumer, nous soulignerons donc relativement à ora argumentatif les points suivants :

I. ora met en relief un nouveau fait comme étant l'information la plus pertinente dans le cadre de l'argumentation, étant donné qu'il actualise l'état informationnel au cours de son déroulement;

2. ora marque une rupture avec le contexte antérieur;

3. cette rupture est accompagnée, en règle générale, par une sorte de contreorientation conférée à l'argumentation;

4. l'effet de contre-orientation ne trouve pas son origine dans la sémantique de ora (qui n'est pas adversative en tant que telle) mais découle plutôt de l'arrangement contextuel. Comme nous l'avons vu dans les exemples proposés dans cette section, les locuteurs jouent sur des contrastes entre des maximes, des principes généraux, des attentes partagées d'un côté, et l'exemplification par le cas particulier, par l'expérience individuelle, par l'exception observée dans le monde réel de l'autre. Tous ces cas particuliers vont à l'encontre des faits et principes établis dans le segment qui précède la proposition introduite par ora.

Si l'on compare maintenant les contextes d'emploi de or français ainsi que de ora italien, on s'aperçoit que or a progressé quant au degré dans lequel l'implicature d'opposition est en train de devenir conventionnelle par rapport à son équivalent italien. Comme nous l'avons vu, en français l'éventail de lectures adversatives est beaucoup plus étendu qu'en italien, où l'accent est surtout mis sur la réorientation de l'argumentation. On peut soulever l'hypothèse selon laquelle la spécialisation de or en tant que marqueur discursif à valeur argumentative (par rapport à maintenant temporel) a contribué à donner plus de relief au potentiel oppositif de or, potentiel qui se voit renforcé grâce à son activation systématique dans des contextes de contraste. Nous avons pu illustrer cette activation systématique à l'aide des exemples [31] à [34]. Dans les exemples que nous avons relevés pour $p$ or $q$, les propositions $p$ et $q$ impliquent des conséquences contraires ( $c$ contraire à $c$, voir [3I]), elles se contredisent nettement ( $q=$ non- $p$ comme en [32] ou $q \rightarrow$ non- $p$ en [33]), ou bien elles ont un rapport antithétique (ce qui est le cas de [34]). Cette tendance qui distingue ora (marque essentiellement de réorientation) et or (avec ces différents degrés de contrastivité) doit être idéalement confirmée par une 
étude approfondie qui se concentre sur la relation quantitative entre les différents emplois (ou bien «lectures») systématiques de or français et de ora italien.

\section{Conclusions}

L'analyse sémantique de certains marqueurs discursifs peut être articulée sur trois niveaux distincts: propositionnel, épistémique-argumentatif et illocutoire (Rossari et al., 2004). Dans ce travail, nous avons proposé une analyse des adverbes ora et maintenant/or de l'italien et du français contemporains, et nous avons montré que ces adverbes peuvent être rapportés à deux de ces trois niveaux: (i) au niveau propositionnel, ora et maintenant sont des adverbes temporels qui, en permettant la localisation des états/événements, contribuent à l'organisation temporelle du discours; (ii) au niveau épistémique-argumentatif, ora et maintenant/or fonctionnent comme des marqueurs qui orientent l'argumentation dans une direction donnée. Le troisième niveau constitué par la fonction illocutoire des adverbes n'a pas été traité ici dans le détail.

Nous avons proposé de décrire comme un trait d'actualisation le trait sémantique qui permet de créer un lien entre les emplois des adverbes à travers ces différents niveaux de représentation. Sur le plan temporel, ora/maintenant signalent l'actualisation du PP de l'énoncé, en l'indexant au dernier TR dans le récit. Au niveau argumentatif, ora/or actualisent l'état informationnel au cours de son déroulement, en mettant en relief un fait asserté comme étant l'information la plus pertinente dans le cadre de l'argumentation.

Comme l'a bien démontré Bertin (200I), or a perdu, au cours de son évolution de l'ancien français au français moderne, ses lectures temporelles. En français moderne, nous observons donc un processus de spécialisation lexicale qui aboutit à une séparation entre le signifié temporel - aujourd'hui réalisé par maintenant - et le signifié épistémique-argumentatif, domaine réservé en exclusivité à or. En italien, ora a réussi à conserver les deux signifiés, ce qui soulève la question que nous avons déjà posée au début de notre article: quel est le rapport entre ora temporel et ora épistémique-argumentatif en italien?

Après notre analyse détaillée, nous sommes maintenant à même de mettre en évidence les traits communs entre les deux lectures, que nous pouvons résumer en trois points distincts.

Tout d'abord, dans les deux lectures temporelle et argumentative, ora révèle son caractère anaphorique en s'orientant vers un repère déjà présent dans le contexte : dans sa lecture temporelle, ora prend le point de référence le plus saillant (qui correspond, en règle générale, au point de localisation établi en dernier) en y établissant le PP à partir duquel la situation est envisagée; dans sa lecture argumentative, ora renvoie à l'état argumentatif établi jusqu'au moment de la saisie qui correspond au fonds commun négocié entre les locuteurs au cours de l'argumentation. 

contradictoire à $p$.

Deuxièmement, un autre trait sémantique en commun consiste dans le fait que ora introduit un PP qui change la perspective en la réorientant d'une façon analogique sur les deux niveaux pris en considération. Ora temporel met en relief un PP qui sépare d'une façon tranchante deux états, un nouvel état qui passe dans le foyer d'attention, et un état antérieur qui passe à l'arrière-plan. La perspective du locuteur passe donc de ce qui a été établi auparavant (c'est-à-dire une perspective orientée vers l'arrière) à une focalisation sur un nouvel état qui se déploie au moment de la saisie, dans une perspective orientée vers l'avant. Ora épistémique-argumentatif met l'accent sur l'introduction d'un argument qui n'a pas encore été pris en considération. Cet argument n'est pas seulement censé actualiser l'état informationnel de l'argumentation, mais il va aussi réorienter le cours de l'argumentation. L'introduction d'un nouvel argument change donc la direction et la perspective de l'argumentation en lui conférant une allure différente, voire inattendue et surprenante. Ce qui relie les deux lectures, la temporelle et l'épistémique-argumentative, c'est la rupture très marquée de l'orientation de la perspective qui passe d'un regard orienté vers l'arrière à une orientation vers l'avant.

Finalement, ora temporel et ora épistémique-argumentatif partagent un trait de saillance, qui se répercute de manière différente sur les deux niveaux en question. Au niveau temporel, ora marque l'éventualité nouvellement introduite en tant qu'état ou événement (possibilité seulement admise en italien) le plus saillant dans le contexte, étant donné qu'il se démarque, par son actualité et sa pertinence, de ce qui a été exposé jusqu'au moment en question. En d'autres termes: puisque ora marque une rupture brusque entre un fait précédent et un «maintenant», ce qui s'est passé auparavant sort du foyer d'attention du locuteur, tandis que la perspective s'ouvre vers ce qui suivra, vers l'avant. Au niveau épistémique-argumentatif, ora marque le contraste entre la direction de l'argumentation telle qu'elle se manifeste dans l'état informationnel établi jusqu'au moment donné et la réorientation de l'argumentation annoncée par ora. L'attention réorientée vers le nouvel argument, accompagnée par un changement de perspective, donne une proéminence à l'argument le plus récent, qui est jugé le plus pertinent, probant et déterminant en ce qui concerne le déroulement de l'argumentation. Comme nous l'avons illustré en 3.I, avec por $q$, la partie ora $q$ peut introduire un nouvel aspect thématique (le cas de la réorientation), infirmer les conséquences de $p$, ou avancer un état des choses qui est contraire ou

Les effets de contraste ne découlent pas de la sémantique (codée dans le signifié) de ora, mais ils sont créés par le contexte qui l'entoure. Cependant, ora s'intègre parfaitement dans un contexte anti-orienté, étant donné qu'il marque une rupture avec le contexte antérieur reliée à un changement de perspective.

Nous pouvons conclure en soulignant une fois de plus que les effets de ora en italien sont moins accentués que ceux de or en français. On peut supposer que le rapport sémantique très étroit (en effet, polysémique) entre ora temporel et ora épistémique-argumentatif est responsable du moindre degré dans lequel cette 
dernière lecture est devenue conventionnelle, étant donné que le signifié d'origine, c'est-à-dire le signifié temporel, plus concret et directement accessible, reste aussi présent dans les emplois épistémiques-argumentatifs. Il en va tout autrement pour maintenant et or en français. Détaché de son signifié étymologique (donc temporel), or, en tant que marqueur de rupture, est systématiquement exploité dans des contextes contrastifs, raison pour laquelle on observe un processus qui vise à rendre les lectures adversatives toujours plus conventionnelles. Ce processus pourrait aboutir à la sémantisation de la lecture adversative, c'est-à-dire à son inclusion dans le noyau sémantique de or. Mais cette perspective n'est qu'une possibilité et, en fin de compte, ce sont les locuteurs qui dans leurs emplois (conventionnels et étendus) de or négocieront le degré dans lequel le potentiel adversatif de cet adverbe devient un trait conventionnel de son emploi.

\section{Références}

Altshuler, D. 2oioa. The Meaning of Now and Other Temporal Locating Adverbs. In M. Aloni et K. Schultz (éd.), Logic, Language and Meaning: I7th Amsterdam Colloquium, Amsterdam, The Netherlands, December 16-I8, 2009, Revised Selected Papers. Lecture Notes in Computer Science 6042. Berlin - Heidelberg - New York: Springer : I83-192.

AltshuleR, D. 20Iob. Temporal Interpretation in Narrative Discourse and Event Internal Reference. Thèse de Doctorat. Rutgers University.

Altshuler, D. 20I4. Discourse Transparency and the Meaning of Temporal Locating Adverbs. Natural Language Semantics 22 (I) : 55-88.

Anscombre, J.-C. et Ducrot, O. 1988. L'argumentation dans la langue. Bruxelles: P. Mardaga.

BANFIELD, A. 1982. Unspeakable Sentences. Narration and Representation in the Language of Fiction. Londres - Boston - Melbourne: Routledge - K. Paul.

Bertin, A. 200I. Maintenant: un cas de grammaticalisation? Langue française 130: 42-64.

Bras, M., Le Draoulec, A. et Asher, N. 2006. Evidence for a Scalar Analysis of Result in SDRT from a Study of the French Temporal Connective Alors. In Proceedings of the SPRIK Conference: "Explicit and Implicit Information in Text. Information Structure accross Languages”. Oslo: Oslo University: 75-79.

De Mulder, W. 2006. Maintenant: un connecteur token-réfléxif? Cabiers Chronos I5: 2I-38.

Ducrot, O. 1980. Les mots du discours. Paris: Minuit.

Ducrot, O. 1984. Le dire et le dit. Paris: Minuit.

Giongi, A. 20I0. About the Speaker. Oxford: Oxford University Press.

Giorgi, A. 20I5. Free Indirect Discourse and the Syntax of the Left Periphery. In J. Guéron (éd.), Sentence and Discourse. Oxford: Oxford University Press.

Giorgi, A. et Pianesi, F. 2004. On the Speaker's and the Subject's Temporal Representation: The Case of the Italian Imperfect. In J. GuÉron et J. LeCARme (éd.), The Syntax of Time. Cambridge: MIT Press: 259-298. 
GuÉron, J. 20I5. Subjectivity and Free Indirect Discourse. In J. GuÉron (éd.), Sentence and Discourse. Oxford: Oxford University Press. Chap. 12: 256-286.

Hunter, J. 2oi2. Now: A Discourse-Based Theory. In M. Aloni, V. Kimmelman, F. Roelofsen, G. W. Sassoon, K. Schulz et M. Westera (éd.), Logic, Language and Meaning: I8th Amsterdam Colloquium, Amsterdam, The Netherlands, December I9-2I, 20II, Revised Selected Papers. Lecture Notes in Computer Science 7218. Berlin Heidelberg - New York: Springer : 37I-38o.

Jouve, D. I990. «Maintenant» et la deixis temporelle. In M.-A. Morel et L. DanonBoileau (éd.), La deixis: colloque en Sorbonne, 8-9 juin 199o. Paris: PUF : 355-363.

Kamp, H. 197I. Formal Properties of Now. Theoria 37 (3): 227-273.

Kamp, H. 2013. Deixis in Discourse. Reichenbach on Temporal Reference [1999]. In K. von Heusinger et A. Ter Meulen (éd.), Meaning and the Dynamics of Interpretation. Selected Papers of Hans Kamp. Leyde - Boston : Brill: I05-I59.

Kamp, H. et Rohrer, C. 1983. Tense in Texts. In R. BäUerle, C. Schwarze, A. von Stechow (éd.), Meaning, Use and Interpretation of Language. Berlin - New York: W. de Gruyter: 250-269.

Kaplan, D. 1989. Demonstratives. An Essay on the Semantics, Logic, Metaphysics, and Epistemology of Demonstratives and Other Indexicals. In J. Almog, J. Perry et H. Wettstein (éd.), Themes from Kaplan. Oxford: Oxford University Press : 48I-563.

KLEIBER, G. 1986. Déictiques, embrayeurs, «token-réflexives», symboles indexicaux, etc. : comment les définir? L'information grammaticale 30 (I): 3-22.

Le Draoulec, A. et Bras, M. 2007. Alors as a Possible Temporal Connective in Discourse. Cabiers Chronos 17: 8I-94.

MÉTrich, R., FAucher, E. et Courdier, G. 1993. Les invariables difficiles. Dictionnaire allemandfrançais des particules, connecteurs, interjections et autres "mots de la communication». Richardménil: Association des Nouveaux cahiers d'allemand. T. I [3 éd. rev. et corr.].

NEF, F. I980. Maintenant $t_{1}$ et maintenant $_{2}$ : sémantique et pragmatique de maintenant temporel et non temporel. In J. David et R. MArtin (éd.), La notion d'aspect. Paris: Klincksieck: $145^{-1}$ - 66 .

Nelken, R. et Francez, N. 1997. Splitting the Reference Time: The Analogy between Nominal and Temporal Anaphora Revisited. Journal of Semantics I4 (4) : 369-416.

NoËL, M. 1996. Un fait de style: maintenant dans Au Château d'Argol de Julien Gracq. Études de linguistique appliquée 102: 157-174.

Partee, B. H. 1984. Nominal and Temporal Anaphora. Linguistics and Philosophy 7 (3): 243-286.

Rossari, C. 2000. Connecteurs et relations de discours: des liens entre cognition et signification. Nancy: Presses universitaires de Nancy.

Rossari, C., Beaulieu-Masson, A. et Cojocari, C. 2004. Autour des connecteurs: réflexions sur l'énonciation et la portée. Bern: P. Lang.

Schlenker, P. H. 2004. Context of Thought and Context of Utterance: A Note on Free Indirect Discourse and the Historical Present. Mind and Language 19: 279-304.

Sharvit, Y. 2008. The Puzzle of Free Indirect Discourse. Linguistics and Philosophy 3I (3): 353-395. 
Smith, C. 1977. The Vagueness of Sentences in Isolation. In W. E. BEACH (éd.), Proceedings of the I3th Regional Meeting of the Chicago Linguistic Society. Chicago: University of Chicago Press: 568-577.

Sмiтh, C. I99I. The Parameter of Aspect. Dordrecht - Boston : Kluwer Academic Publishers.

Sмiтh, C. 1997. The Parameter of Aspect. Dordrecht - Boston : Kluwer Academic Publishers [ $2^{\mathrm{e}}$ éd. rev. et corr.].

\section{Corpus}

ATILF (laboratoire Analyse et traitement informatique de la langue française). Base textuelle Frantext. En ligne à l'adresse suivante: http://www.frantext.fr/.

Corpus CORIS. En ligne à l'adresse suivante: http://corpora.ficlit.unibo.it/coris_eng.html. Corpus «La Repubblica». En ligne à l'adresse suivante: http://sslmit.unibo.it/repubblica. Le Monde sur CD-ROM: 200I-2002. Paris: Le Monde diplomatique. 